University of Rhode Island

DigitalCommons@URI

Civil \& Environmental Engineering Faculty

Publications

Civil \& Environmental Engineering

$4-28-2020$

\title{
Fracture toughness of sodium aluminosilicate hydrate (NASH) gels: Insights from molecular dynamics simulations
}

Gideon A. Lyngdoh

Sumeru Nayak

Rajesh Kumar

N. M. Anoop Krishnan

Sumanta Das

University of Rhode Island, sumanta_das@uri.edu

Follow this and additional works at: https://digitalcommons.uri.edu/cve_facpubs

The University of Rhode Island Faculty have made this article openly available.

Please let us know how Open Access to this research benefits you.

This is a pre-publication author manuscript of the final, published article.

Terms of Use

This article is made available under the terms and conditions applicable towards Open Access Policy Articles, as set forth in our Terms of Use.

\section{Citation/Publisher Attribution}

G.A. Lyngdoh, S. Nayak, R. Kumar, N.M. Anoop Krishnan, S. Das, Fracture toughness of sodium aluminosilicate hydrate (NASH) gels: Insights from molecular dynamics simulations, Journal of Applied Physics. 127 (2020) 165107. https://doi.org/10.1063/1.5144876.

This Article is brought to you for free and open access by the Civil \& Environmental Engineering at DigitalCommons@URI. It has been accepted for inclusion in Civil \& Environmental Engineering Faculty Publications by an authorized administrator of DigitalCommons@URI. For more information, please contact digitalcommonsgroup@uri.edu. 


\title{
Fracture Toughness of Sodium Aluminosilicate Hydrate (NASH) Gels: Insights from Molecular Dynamics Simulations
}

\author{
Gideon A. Lyngdoh ${ }^{1}$, Sumeru Nayak ${ }^{1}$, Rajesh Kumar², N.M. Anoop Krishnan ${ }^{2,3}{ }^{*}$, and Sumanta \\ $\operatorname{Das}^{1, *}$ \\ ${ }^{1}$ Department of Civil and Environmental Engineering, University of Rhode Island, Kingston, RI, \\ 02881, USA \\ ${ }^{2}$ Department of Civil Engineering, Indian Institute of Technology Delhi, Hauz Khas, New Delhi, \\ 110016, India \\ ${ }^{3}$ Department of Materials Science and Engineering, Indian Institute of Technology Delhi, Hauz \\ Khas, New Delhi, 110016, India \\ *Corresponding authors: N. M. A. Krishnan (krishnan@iitd.ac.in), S. Das (sumanta das@uri.edu)
}

\section{Journal of Applied Physics 127, 165107 (2020); \\ https://doi.org/10.1063/1.5144876}

\begin{abstract}
This paper evaluates fracture toughness of sodium aluminosilicate hydrate (N-A-S-H) gel formed through alkaline activation of fly ash via molecular dynamics simulations. The short-and medium-range order of constructed $\mathrm{N}-\mathrm{A}-\mathrm{S}-\mathrm{H}$ structures shows good correlation with the experimental observations signifying the viability of the $\mathrm{N}-\mathrm{A}-\mathrm{S}-\mathrm{H}$ structures. The simulated fracture toughness values of $\mathrm{N}-\mathrm{A}-\mathrm{S}-\mathrm{H}(0.4-0.45$ $M P a m^{0.5}$ ) appears to be of the same order as the available experimental values for fly ash-based geopolymer mortars and concretes. These results suggest the efficacy of the molecular dynamics simulation towards obtaining realistic fracture toughness of $\mathrm{N}-\mathrm{A}-\mathrm{S}-\mathrm{H}$ which is otherwise very challenging to obtain experimentally and no direct experimental fracture toughness values are yet available. To further assess the fracture behavior of N-A-S-H, number of chemical bonds formed/broken during elongation and their relative sensitivity to crack growth are evaluated. Overall, the fracture toughness of $\mathrm{N}-\mathrm{A}-\mathrm{S}-\mathrm{H}$ presented in this paper paves the way for multiscale simulation-based design of tougher geopolymers.

Keywords: fly ash; geopolymer; sodium aluminosilicate hydrate gel; fracture toughness; molecular dynamics simulations
\end{abstract}




\section{INTRODUCTION}

Concrete is considered as one of the most widely used construction materials in the world ${ }^{1}$. However, the production of cement is associated with significant carbon footprint and it contributes to $5-7 \%$ of the global $\mathrm{CO}_{2}$-emission ${ }^{2-4}$. With a view to provide a sustainable alternative to ordinary Portland cement (OPC), geopolymer binders have been proposed ${ }^{5-11}$. Geopolymer binders are produced through alkaline activation of aluminum or silicon rich materials (such as fly ash or Metakaolin) ${ }^{5-11}$. The most common activating agents, used in synthesis of such geopolymer binders, are Alkali (Na or K) hydroxides and/or silicates $^{7,9-11}$. Geopolymers have been shown to exhibit superior mechanical performance and better durability against chlorides/sulfate attack as compared to OPC $\mathrm{OP}^{9,10,12,13}$. Additionally, it has been shown to reduce the $\mathrm{CO}_{2}$ emissions by $25^{\sim} 40 \%$,10,11,14 when compared to OPC binder. Such enhanced credentials of geopolymer binders in terms of both reduced carbon footprint and improved mechanical/durability performance makes them very alluring environment-friendly alternative to OPC. However, similar to OPC binder, geopolymers are also inherently brittle and shows poor fracture resistance ${ }^{15}$. Hence, any improvement in the fracture resistance of these binders would contribute towards enhancement in their longevity and sustainability.

Fundamental design of geopolymer binders for enhanced fracture toughness based on bottom-up approach ${ }^{16}$ would necessitate intrinsic fracture toughness of the main binding phase in the geopolymer binders (such as sodium-aluminosilicate-hydrate (N-A-S-H) gel formed through alkaline activation of fly ash by $\mathrm{NaOH}$ ) which remains largely unknown so far. Highly heterogeneous nature of such geopolymer binders makes it challenging to extract such intrinsic fracture response of the main binding phase. Further, although fly ash-based geopolymers have been researched extensively ${ }^{17-19}$ over the past four decades, the molecular structure of N-A-S-H has recently been proposed ${ }^{20-25}$. As such, a fundamental investigation using atomistic simulations, to study the fracture response of $\mathrm{N}-\mathrm{A}-\mathrm{S}-\mathrm{H}$ remains an open problem.

Towards that end, this paper reports the simulated fracture toughness of N-A-S-H obtained from atomic scale computed using molecular dynamics (MD) simulations. The molecular structure of N-A-S-H gel is developed by following the general procedure to obtain an amorphous glass from crystalline structure i.e., melt-quench approach. The obtained glassy structure is hydrated by performing Grand Canonical Monte Carlo (GCMC) simulation ${ }^{26}$. The inter-atomic interactions are modeled using a reactive force field $(\text { ReaxFF })^{27}$, which is parametrized for elements such as $\mathrm{Na}, \mathrm{Al}, \mathrm{Si}, \mathrm{O}$, and $\mathrm{H}^{23}$. In order to evaluate the

influence of water content on the fracture response of $\mathrm{N}-\mathrm{A}-\mathrm{S}-\mathrm{H}$, several molecular structures with varying water content are constructed. The viability of the constructed $\mathrm{N}-\mathrm{A}-\mathrm{S}-\mathrm{H}$ structures are validated against 
experimental neutron diffraction data ${ }^{28-32}$. Overall, the fracture toughness of N-A-S-H gel reported in this paper paves the way for multiscale numerical simulation-based design of fly ash-based geopolymer binders for enhanced performance.

\section{SIMULATION METHODOLOGY}

\subsection{Preparation of the N-A-S-H model}

The model construction procedure is schematically depicted in Figure 1.

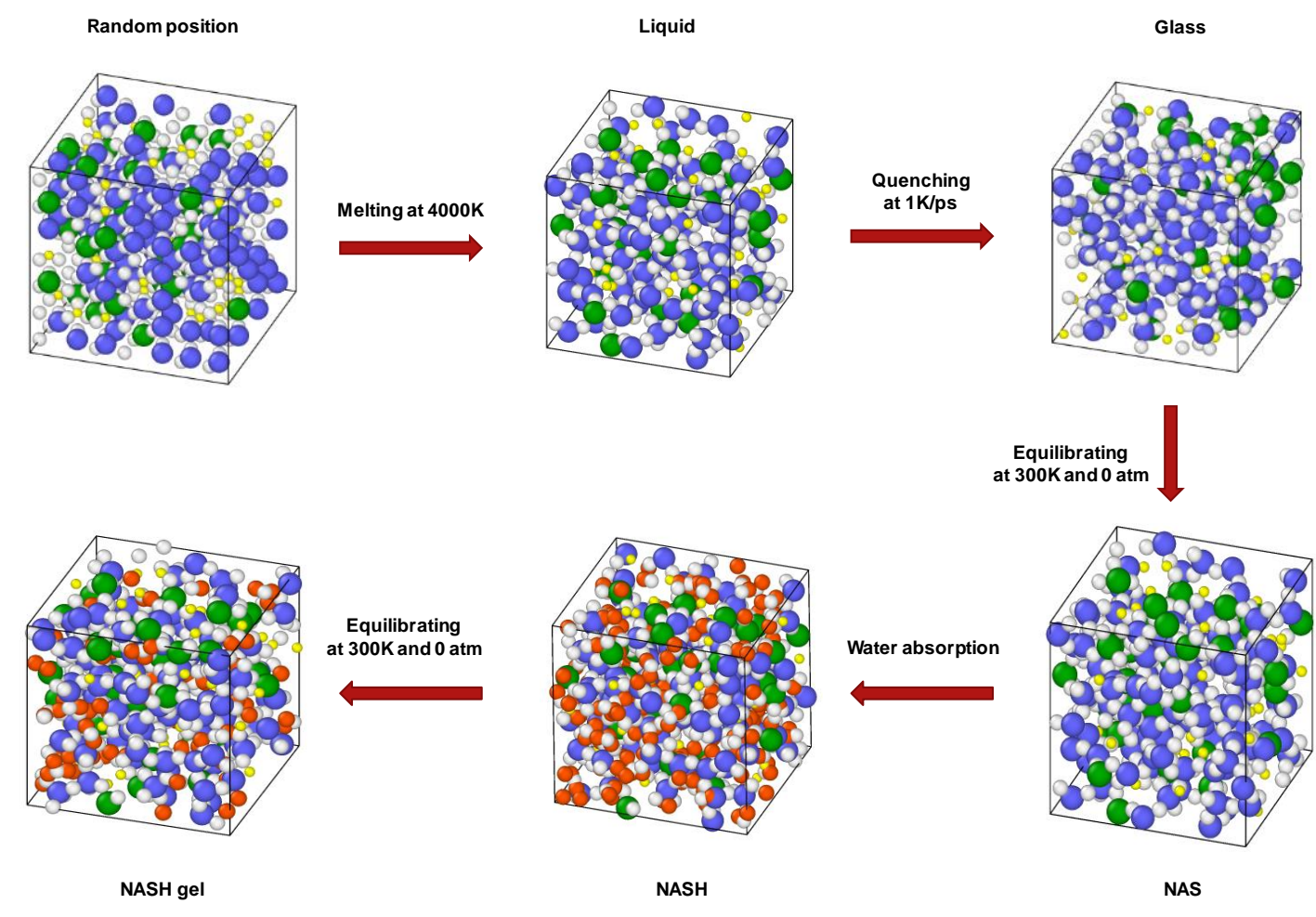

Figure 1: Schematic representation of the model construction procedure (Colors scheme - Al: Green, Si: Blue, Na: Yellow, O: White, and $\mathrm{H}$ : Red)

First, the initial structure of sodium aluminosilicate (NAS) glass (which is approximately 4000 atoms) is prepared by randomly placing the atoms $(\mathrm{Na}, \mathrm{Al}, \mathrm{Si}$, and $\mathrm{O})$ in a bounded box with a minimum distance of $2 \AA$ between each atom. In this study, the glass composition is prepared with Si/Al ratio equal to 3 . To maintain the charge neutrality in the structure $\mathrm{Na} / \mathrm{Al}$ ratio is kept as unity. Here the role of $\mathrm{Na}^{+}$cation is to neutralize the negative charge due to the formation of $\left[\mathrm{AlO}_{4}\right]^{-1}$. The structure is initially melted at $4000 \mathrm{~K}$ in both NPT ensemble at zero pressure for $400 \mathrm{ps}$ and NVT ensemble for another $400 \mathrm{ps}$ in order to confirm the loss of memory of the initial configuration. A time step of $0.5 \mathrm{fs}$ is adopted for both the ensembles. The MD simulation is carried out using an open-source code LAMMPS ${ }^{33}$. The inter-atomic interactions are 
modelled by using a reactive forcefield (ReaxFF) ${ }^{23}$ along with the charge equilibration method. A tolerance of $10^{-6} \mathrm{kcal} / \mathrm{mol}$ is adopted during the charge equilibration. The temperature is linearly decreased from $4000 \mathrm{~K}$ to $300 \mathrm{~K}$ at the cooling rate of $1 \mathrm{~K} / \mathrm{ps}$. Such cooling rate has also been adopted successfully for quenching of silicate glasses ${ }^{34-37}$. The obtained glassy structure is then equilibrated for $400 \mathrm{ps}$ in NPT emsemble at zero pressure and 300K temperature followed by NVT emsemble for another 200 ps before running for production. The final dimension of the N-A-S glass after quenching and relaxation at 300K and 0 atm pressure is $34 \AA \times 34 \AA \times 34 \AA$. The MD simulation is carried out considering periodic boundary condition (PBC) along all three directions. To hydrate the glass structure, a well-established method Grand Canonical Monte Carlo (GCMC) simulation ${ }^{26}$ in the grand canonical ensemble $(\mu V T)$ is adopted. In this study, a value for chemical potential $\mu$ is set as $0 \mathrm{eV}$ and the temperature is maintained equal to the system at $300 \mathrm{~K}$ to provide unlimited supply of water. To allow saturation with a stable distribution of water, the simulation is run for one million steps (as shown in Figure 2).

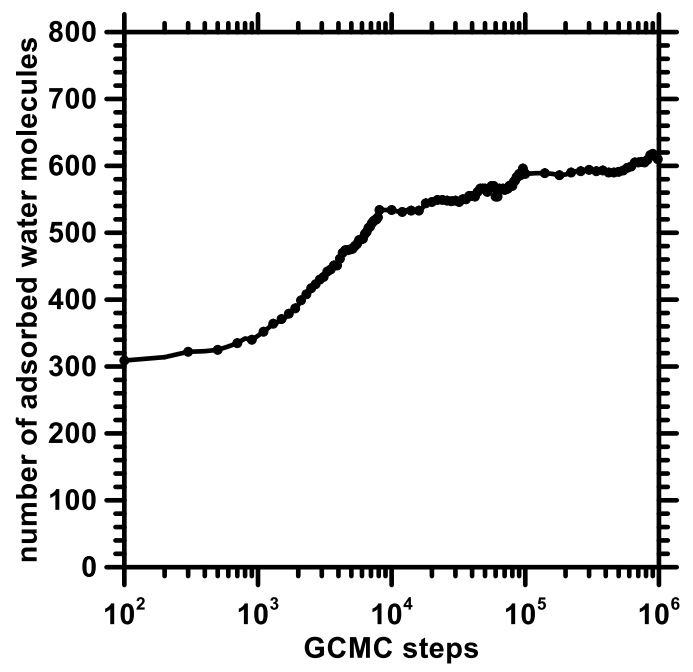

Figure 2: Number of water molecules adsorbed with increasing GCMC steps

In this study, five different water contents $(5 \%, 10 \%, 15 \%$ and $20 \%$ by weight percentage) in the N-A-S-H structure are considered based on water contents reported in the literature $22,38,39$. Variations in water content is achieved by saving the configuration of the structure during GCMC at different intervals. It is noteworthy that the N-A-S-H structure is saturated at water content equal to $20 \%$ and hence a maximum water content of $20 \%$ is considered in this paper. Similar realistic range of water content is reported in an experimental study by Ly et al ${ }^{38}$. Here onwards, N-A-S-H structures with $5 \%, 10 \%, 15 \%$, and $20 \%$ water content are donated as NASH5, NASH10, NASH15, and NASH20 respectively. All simulations are 
performed in an open software code LAMMPS $^{33}$. For inter-atomic interactions, ReaxFF potential ${ }^{23}$ is used along with the charge equilibration method ${ }^{40}$. In this study, a tolerance of $10^{-6} \mathrm{kcal} / \mathrm{mol}$ is adopted during the charge equilibration. The thermodynamic properties are integrated using Verlet method ${ }^{41}$ and a timestep equal to $0.5 \mathrm{fs}$ is adopted. The constructed representative atomic structure of $\mathrm{N}-\mathrm{A}-\mathrm{S}-\mathrm{H}$ containing $15 \%$ water is shown in Figure 3. The chemical composition for all the N-A-S-H structures and its water content is shown in Table 1 along with obtained density. The computed density from MD simulation for $\mathrm{N}-\mathrm{A}-\mathrm{S}-\mathrm{H}$ structure lies in the range from $1.9-2.24 \mathrm{~g} / \mathrm{cm}^{3}$, which is in line with the experimental values (1.8$2.4 \mathrm{~g} / \mathrm{cm}^{3}$ ) reported in the literature ${ }^{38,42}$.

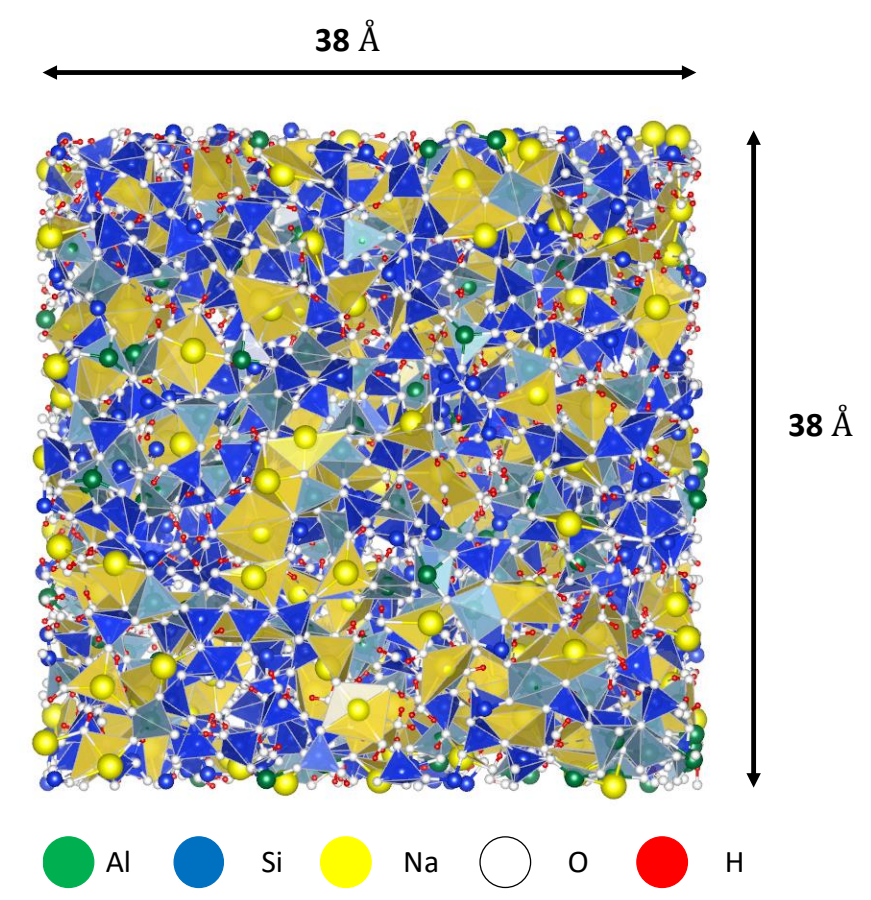

Figure 3: Representative N-A-S-H structure containing $15 \%$ water

Table 1: Chemical composition and density of constructed NAS glass and N-A-S-H structures

\begin{tabular}{|c|c|c|c|c|c|c|c|c|c|}
\hline \multirow{2}{*}{ Structure } & \multicolumn{2}{|c|}{$\mathrm{SiO}_{2}$} & \multicolumn{2}{|c|}{$\mathrm{Al}_{2} \mathrm{O}_{3}$} & \multicolumn{2}{|c|}{$\mathrm{Na}_{2} \mathrm{O}$} & \multicolumn{2}{|c|}{ Water content } & \multirow{2}{*}{$\begin{array}{l}\text { Density } \\
\left(\mathrm{g} / \mathrm{cm}^{3}\right)\end{array}$} \\
\hline & Wt \% & $\mathrm{mol} \%$ & Wt \% & $\mathrm{mol} \%$ & Wt \% & $\mathrm{mol} \%$ & Wt \% & $\mathrm{mol} \%$ & \\
\hline NAS & 68.74 & 75 & 19.44 & 12.5 & 11.82 & 12.5 & 0.00 & 0 & 2.24 \\
\hline \multirow{4}{*}{$\mathrm{N}-\mathrm{A}-\mathrm{S}-\mathrm{H}$} & 65.26 & 62.82 & 18.46 & 10.47 & 11.22 & 10.47 & 5.06 & 16.24 & 2.27 \\
\hline & 61.56 & 52.65 & 17.41 & 8.77 & 10.58 & 8.77 & 10.45 & 29.81 & 2.28 \\
\hline & 58.44 & 45.7 & 16.53 & 7.62 & 10.05 & 7.62 & 14.98 & 39.07 & 2.16 \\
\hline & 55.44 & 39.72 & 15.41 & 6.51 & 9.37 & 6.51 & 19.78 & 47.27 & 1.95 \\
\hline
\end{tabular}




\subsection{Structural characterization}

Structural characterization is performed and validate with the experimental data from literatures in order to evaluate the viability of the constructed N-A-S-H structures. In this paper, the structural characterization of the constructed N-A-S-H models is performed in terms of both short and mediumrange order. While short-range order is characterized using pair distribution function, structure factor is used to quantify the medium-range order which are described in the forthcoming sub-sections.

\subsubsection{Pair distribution function}

To validate the structural properties of the generated structure with the available experimental data, short-range neutron pair distribution function (PDF) is computed as follow ${ }^{35,43-46}$ :

$$
g_{N}(r)=\frac{1}{\sum c_{i} c_{j} b_{i} b_{j}} \sum c_{i} c_{j} b_{i} b_{j} g_{i j}(r)
$$

where $c_{i}$ is the fraction of $i$ atoms $(i=\mathrm{Al}, \mathrm{Si}, \mathrm{Na}$, or $\mathrm{O}), b_{i}$ is the neutron scattering length of the species, and $g_{i j}$ are the partial PDFs. Using the partial PDF, coordination number of each of the species can be obtained by computing the number of neighbors within the first coordination shell of the respective atoms. The cutoff distance is taken from the first minimum of the respective partial PDFs. The PDF is mainly applicable for investigating the properties of the structure in short range $\operatorname{order}(<3 \AA)$.

\subsubsection{Structure Factor}

The structure factor is adopted here to evaluate the medium-range properties of the atomic structure. The partial structure factors are calculated from Fourier transformation of the partial PDF $g_{i j}(r)$ as shown in Equation 1

$$
S_{i j}(Q)=1+\rho_{0} \int 4 \pi r^{2}\left[g_{i j}(r)-1\right]\left(\frac{\sin \left(Q_{r}\right)}{Q_{r}}\right)\left(\frac{\sin \left(\frac{\pi r}{R}\right)}{\frac{\pi r}{R}}\right) d r
$$

Where $Q$ is the scattering vector, $\rho_{0}$ is the average atom number density, and $R$ is the maximum value of the integration in real space, which is set to half of the size of one side of the simulation cell. Here, the Lorch-type window function is used in order to reduce the effect of the finite cut-off radius in the integration ${ }^{47}$. While the use of such window type has shown to reduce the ripples at low $Q$, note that this may induce some broadening of the structure factor peaks ${ }^{48}$. Such window function has also been applied successfully to silicate glasses using MD simulation ${ }^{35,46}$. The total structure factor is calculated as ${ }^{35,43-46}$ :

$$
S_{N}(Q)=\frac{1}{\sum c_{j} c_{i} b_{j} b_{i}} \sum c_{i} c_{j} b_{i} b_{j} S_{i j}(Q)
$$


where $c_{i}$ and $c_{j}$ are the fractions of atoms and $b_{i}$ and $b_{j}$ are neutron scattering lengths, for elements $i$ and $j$, respectively. The partial PDF and structure factor are plotted by taking statistical average over 100 frames at $300 \mathrm{~K}$.

\subsection{Methodology to predict fracture toughness}

The fracture response of the constructed N-A-S-H structures is evaluated using the methodology explained in this section hereafter. To obtain the fracture response of the $\mathrm{N}-\mathrm{A}-\mathrm{S}-\mathrm{H}$, tensile tests are simulated by applying uniaxial strain on the constructed N-A-S-H structures (containing pre-existing flaw). For fracture simulations of N-A-S-H structures, a bounding box of size $114 \AA$ x $76 \AA$ x $38 \AA$ is constructed which contains a pre-existing crack of size $36 \AA$ × $8 \AA$ x $38 \AA$. In the present work, the uniaxial tensile deformation through progressive elongation at a strain rate of $0.001 \mathrm{ps}^{-1}\left(\right.$ or $\left.10^{9} \mathrm{~s}^{-1}\right)$ is adopted with a time step of $0.5 \mathrm{fs}$, which is considered to be low enough for atomic restructuring mechanics to takes place in the stressed system, while maintaining computational tractability ${ }^{20,49}$. Similar strain rates are also adopted in MD simulations of similar materials ${ }^{21,50-52}$. The stress value is calculated by using the virial theorem and the respective stress values are obtained by averaging when running at $300 \mathrm{~K}$ for every 100 time steps. Here, the simulation is performed in plane strain condition where no movement is allowed in the $X$ and $Z$ directions (i.e., stresses in these directions are not equal to zero).

Under uniaxial strain, the stress is calculated using the virial stress approach ${ }^{53,54}$ :

$$
\sigma_{i j}^{V}=-\frac{1}{V} \sum_{\alpha=1}^{N}\left[m_{\alpha} v_{i}^{\alpha} v_{j}^{\alpha}+\frac{1}{2} \sum_{\beta \neq \alpha} r_{i j}^{\alpha \beta} f_{i j}^{\alpha \beta}\right]
$$

where the terms $\mathrm{V}$ represents the volume of bounding box, $\mathrm{N}$ is the number of atoms in the model, $\mathrm{m}$ is the mass of atom, $v$ is the velocity of atom, $r$ is the interatomic distance, and $f$ is the interatomic force. The subscript $i, j$ takes the value 1,2 , and 3 for $\mathrm{X}, \mathrm{Y}$, and $\mathrm{Z}$ directions respectively. The above virial stress formula corresponds to the true stress, and in order to obtain the engineering stress, the virial stress is scaled by the initial cross-section area of the model. The engineering strain and stress are thus defined by using the following formula:

$$
\begin{gathered}
\sigma=\frac{\sigma_{i j}^{V} A}{A_{o}} \\
\varepsilon=\frac{L-L_{o}}{L_{o}}
\end{gathered}
$$

where $A$ and $L$ are the instantaneous cross-sectional area and current length, and $A_{o}$ and $L_{o}$ are the initial cross-sectional area and length of the simulated box respectively. 
To evaluate the fracture properties from MD simulation, the critical strain energy release rate $\left(G_{c}\right)$ is derived based on the energetic theory of fracture mechanism ${ }^{55-58}$, and the thermodynamics integration is imposed during crack propagation. The advantage of this approach is that no assumption is adopted on the mechanical behavior of the material during fracture, and hence it is applicable to both brittle ${ }^{55}$ and ductile material ${ }^{44,59}$. In this approach, when the crack is initialed and propagated through the structure, the energy is released in the form of fracture energy. The fracture energy is correlated to the critical energy release rate $\left(G_{c}\right)$ which correspond to the energy released per unit area of crack propagated. The critical energy release rate can be written as the ratio of the free energy of the system $(\Delta F)$ with respect to the total surface area created at the end of the fracture $(\Delta A)$. In the work by Brochard et al. ${ }^{55}$, the fracture energy of materials is calculated by employing thermodynamic integration which is integration of $\sigma_{y}$ over the whole process (i.e., the external work) as shown in Eq. (7). This formulation is based on the energetic theory of fracture mechanics ${ }^{56-58}$.

$$
G_{c}=\frac{\Delta F}{\Delta A}=\frac{L_{x} L_{z}}{\Delta A} \int_{L_{y 0}}^{L_{y \max }} \sigma_{y} d L_{y}
$$

where $\Delta A$ is the total area created due to fracture when the crack surface is fully propagated, $\sigma_{y}$ is the average stress due to applied uniaxial tensile strain in y direction, and $L_{x}, L_{y}$ and $L_{z}$ are the dimension of the bounding box in $\mathrm{x}, \mathrm{y}$ and $\mathrm{z}$ directions respectively. $y_{0}$ and $y_{\max }$ correspond to the initial dimension along y axis before application of strain and final dimension when the crack is fully propagated, respectively. The above formulation can be directly correlated to the Griffith theory of fracture ${ }^{56}$. The relationship between critical stress intensity factor $\left(K_{I C}\right)$ and $G_{C}$ for isotropic materials as per the Irwin's formula $a^{57,60}$ is given as:

$$
K_{I C}=\sqrt{\frac{G_{C} E}{1-v^{2}}}
$$

where $E$ is the Young's modulus of the material, and $v$ is the Poisson's ratio. To obtain the Young's modulus and Poisson's ratio of NASH gel, a tensile deformation is performed to each respective structure without cracks using the same strain rate of $0.001 \mathrm{ps}^{-1}$. The representative dimensions of the respective NASH structures with varying water content are $38 \AA \times 38 \AA \times 38 \AA$. A linear fitting of stress-strain plot within $1 \%$ strain is carried out to calculate the Young's modulus for each uniaxial direction and average value is used in the calculation. The Poisson's ratio is obtained by taking the ratio of lateral strain with 
respect to tensile strain. Similar methodology has been successfully implemented towards MD-based fracture toughness prediction of calcium silicate hydrate (CSH) by Bauchy et al. ${ }^{44}$.

\section{SIMULATION RESULTS AND DISCUSSIONS}

\subsection{Structural characterization: Neutron pair distribution function and neutron structure factor}

The short-range distribution is first investigated by computing the total pair distribution function (PDF) using Equation 1. Figure 4(a) shows a comparison of the pair distribution function of N-A-S-H structure with different water content against the available experimental data ${ }^{32} u s i n g$ neutron diffraction. In the experimental study, the geopolymer paste was prepared by activating pure metakaolin with deuterated sodium silicate solution with molar ratio $\mathrm{SiO}_{2} / \mathrm{Na}_{2} \mathrm{O}$ equal to $2^{32}$. While the first peak at $1.0 \AA$ in the experimental PDF corresponds to the D-O interaction ( $D$ refers to deuterium where heavy water is used in order to prepare the geopolymer binder in the experimental study ${ }^{32}$ ), the same peak for simulated structures indicates the $\mathrm{H}-\mathrm{O}$ interaction. The $\mathrm{Si}-\mathrm{O}$ peak value of $1.62 \AA$ corresponds to the $\mathrm{Si}-\mathrm{O}$ bond length of Si tetrahedral. For the case of $\mathrm{Al}-\mathrm{O}$, the peak is less clear as they arise from the superposition of different coordination number $(\mathrm{CN})$ of $\mathrm{Al}(\mathrm{CN}=4,5)$. The other peak value at $2.6 \AA$ corresponds to the $0-\mathrm{O}$ interactions. All these peaks (in the simulated PDFs) are in excellent agreement with experimental data ${ }^{32}$.
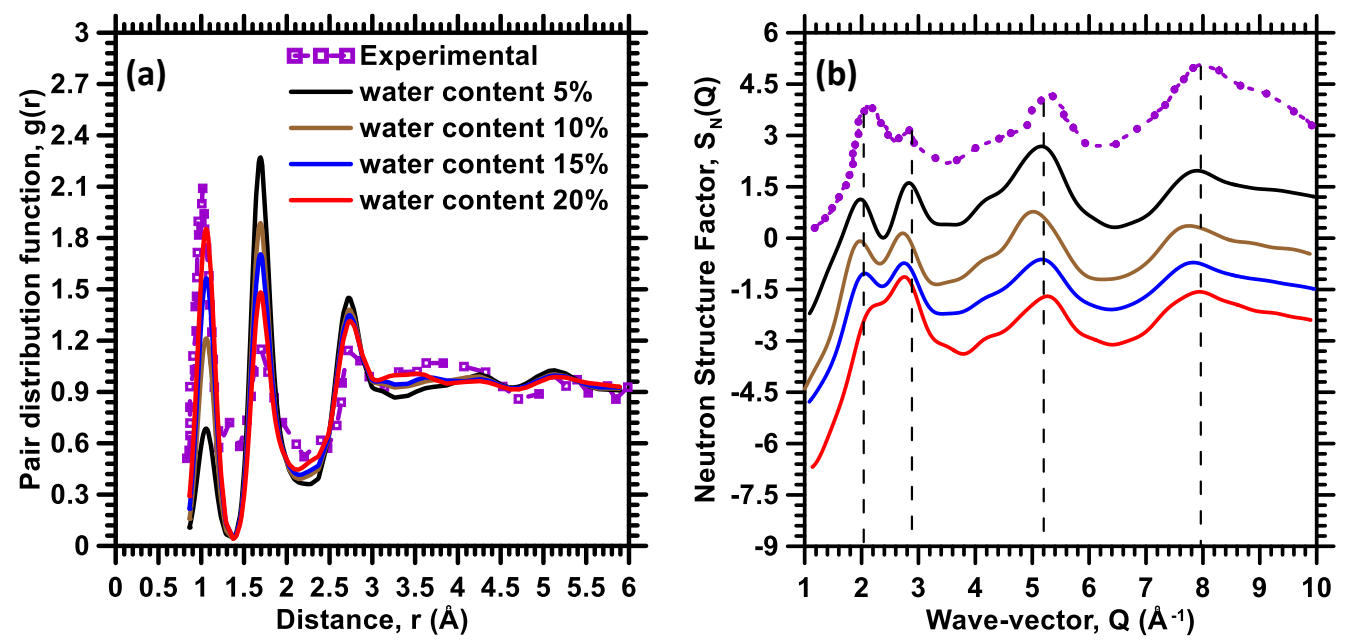

Figure 4. (a) Pair distribution function and (b) structure factor of the N-A-S-H model, compared with the experimental data

To shed more light on the comparison between the experimental and simulated PDFs, the Wright factor is computed to compare the degree of agreement between the computed pair distribution function (PDF) and the experimental data for both the proposed structure and the structure from the previous study. The Wright factor is expressed $a s^{61}$ : 


$$
\mathcal{R}_{\mathcal{H}}=\left[\frac{\sum_{i=1}^{n}\left(g\left(r_{i}\right)-g_{\exp }\left(r_{i}\right)\right)^{2}}{\sum_{i=1}^{n}\left(g_{\exp }\left(r_{i}\right)\right)^{2}}\right]
$$

where $g_{\text {exp }}\left(r_{i}\right)$ is the experimental total PDF. These factors are calculated over the range from $1 \AA$ to 10 $\AA$ And the computed $\mathcal{R}_{\mathcal{H}}$ for NASH with water content of $5 \%, 10 \%, 15 \%$ and $20 \%$ are $25.5 \%, 18.9 \%, 11.9 \%$ and $7.82 \%$, respectively. Since the value of $\mathcal{R}_{\mathcal{H}}$ below $12 \%$ can be considered as a good agreement ${ }^{35}$, this implies that NASH structures with water contents in the range of $15-20 \%$ represents the realistic water content in the NASH gel. To investigate the medium range order of the N-A-S-H structures, structure factors are computed using Equation 2 and Equation 3. Figure 4(b) plots the simulated and experimental neutron structure factors. It is clearly seen that the four distinct peaks in the simulated structure factor plots are well-correlated with the experimental data. The position of the first sharp diffraction peak (FSDP) matches with the experimental data signifying the good correlation in the medium range structure. Based on the good agreement between the experimental and simulated structural characteristics both in the short- and medium-range order, a realistic water content in the range of $15-20 \%$ is considered hereafter in the forthcoming sections.

\subsection{Structural Polymerization}

In this section, the structural polymerization is assessed by calculating the $\mathrm{Q}_{\mathrm{n}}$ distribution for $\mathrm{N}-\mathrm{A}-\mathrm{S}-\mathrm{H}$ with different water content. The $Q_{n}$ distribution is based on the tetrahedral network formed by Si and Al elements (where $\mathrm{n}$ is the number of bridging oxygen) as shown in Figure $5(\mathrm{a}-\mathrm{b})$. Figure $5(\mathrm{a})$ shows the $\mathrm{Q}_{\mathrm{n}}$ distribution of $\mathrm{Si}$. It is evident that with increase in water content the $Q_{4}$ decreases whereas the $Q_{3}$ and $\mathrm{Q}_{2}$ increases which indicates depolymerization of the structure with increase in water content. Similar behavior is also observed for $\mathrm{Al}$, where the $Q_{4}$ and $Q_{5}$ decreases and $Q_{3}$ increases with increase in water content. It is observed that in the N-A-S-H gel, the water molecules help in stretching the Si-O-T (T=Si, Al) bond which results in the separation between the tetrahedral $\mathrm{SiO}_{4}$ units and nearby $\mathrm{AlO}_{4} / \mathrm{AlO}_{5}$ units ${ }^{62}$. 

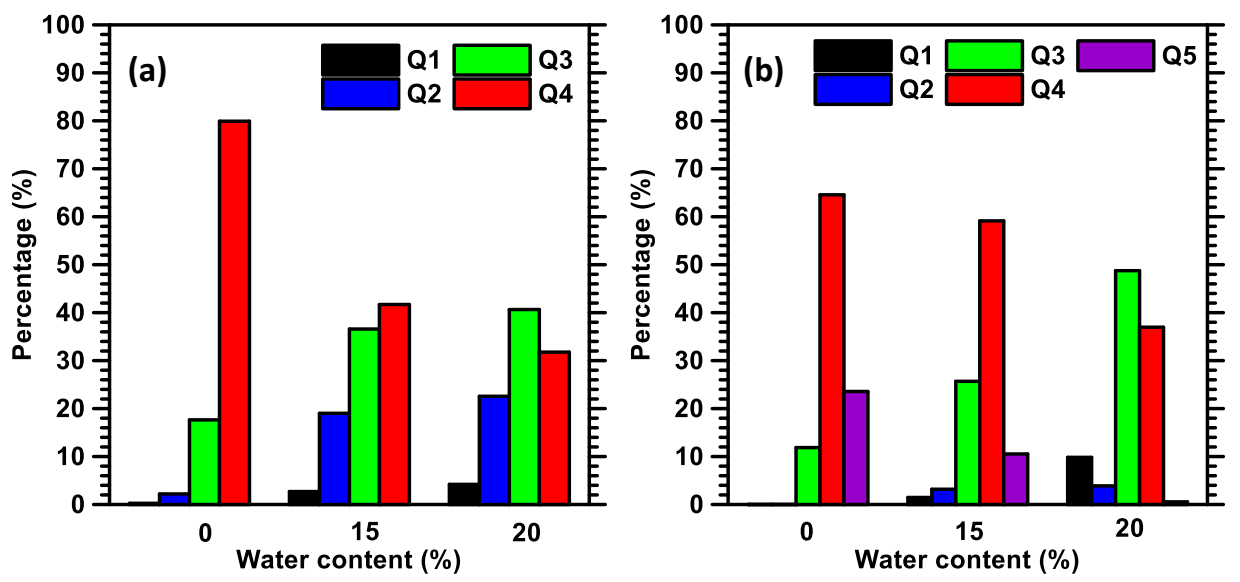

Figure 5: $Q_{n}$ distribution for (a) Si and (b) Al in NASH structures containing $15 \%$ and $20 \%$ water

\subsection{Fracture toughness of $\mathrm{N}-\mathrm{A}-\mathrm{S}-\mathrm{H}$}

With a view to evaluate the fracture toughness prediction capability of the aforementioned simulation methodology, fracture toughness of sodium aluminosilicate (NAS) glass is first computed and compared against the experimental value available in the literature. To evaluate fracture response of NAS glass, a rectangular initial crack is first incorporated in the NAS molecular structure as shown in Figure 6.

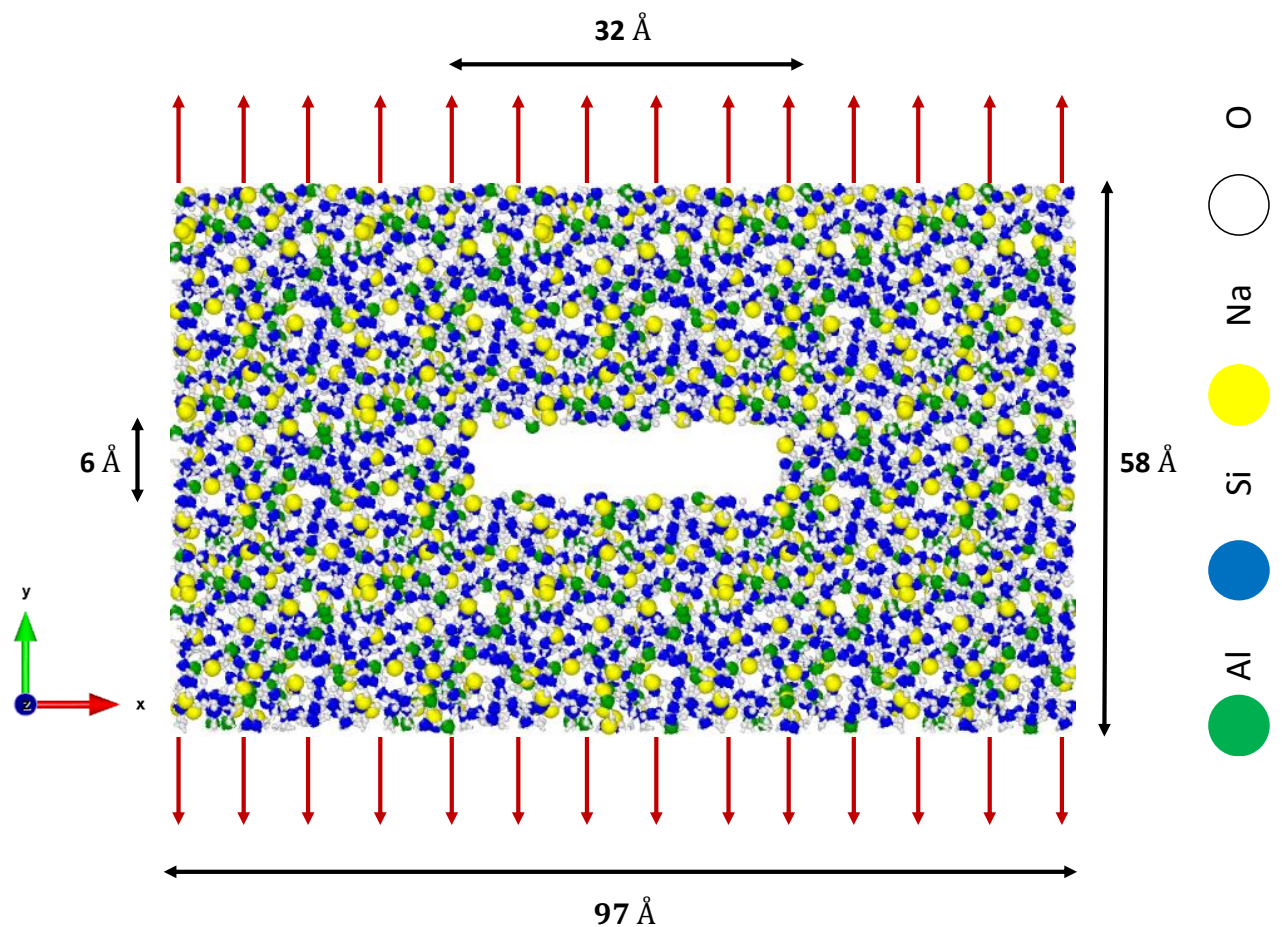

Figure 6. Constructed NAS glass structure with initial crack. 
The crack is formed by removing the atoms located inside the rectangular volume along the $z$ direction (out of plane direction). The crack area is chosen such that the length of the crack in the $x$ direction is at least five times larger than the width in the $y$ direction, thus leading to stress concentration towards $\mathrm{x}$ direction. Such volume of crack with respect to the total volume of bounding box of the molecular structure has been shown to maintain stability in the literature ${ }^{34}$. In this work, a crack/box ratio of 0.32 is maintained in the x-direction. While the bounding box of the periodic molecular structure is of size $97 \AA$ $x 58 \AA$ x $20 \AA$, the initial crack dimension for the NAS glass is approximately $32 \AA$ x $6 \AA$ x $20 \AA$. Note that, while crack length can have significant effect on maximum stress achieved for the system, fracture energy is found to be independent of the crack length or system size using the present methodology ${ }^{63,64}$. A detailed analysis of system size and crack length effects has been done by Brochard et $\mathrm{al}^{63}$ for materials with and without plastic deformations. Prior to any deformation in the system, the system is first relaxed using energy minimization. The tensile strain is then applied in the direction perpendicular to the initial crack. The tensile strain is increased until the crack is fully propagated. The entire fracture simulation is performed in NVT ensemble, where the temperature is controlled by a Nose-Hoover thermostat ${ }^{65,66}$. Figure 7 shows the simulated tensile stress-strain response of the NAS glass. The simulated stress-strain plot shows linear elastic behavior till a strain of $15 \%$. During this stage, there is no crack propagation and the energy developed is stored in the form of mechanical elastic energy only. As the deformation is further increased, the crack starts to propagate and there is a sudden drop in the stress value.

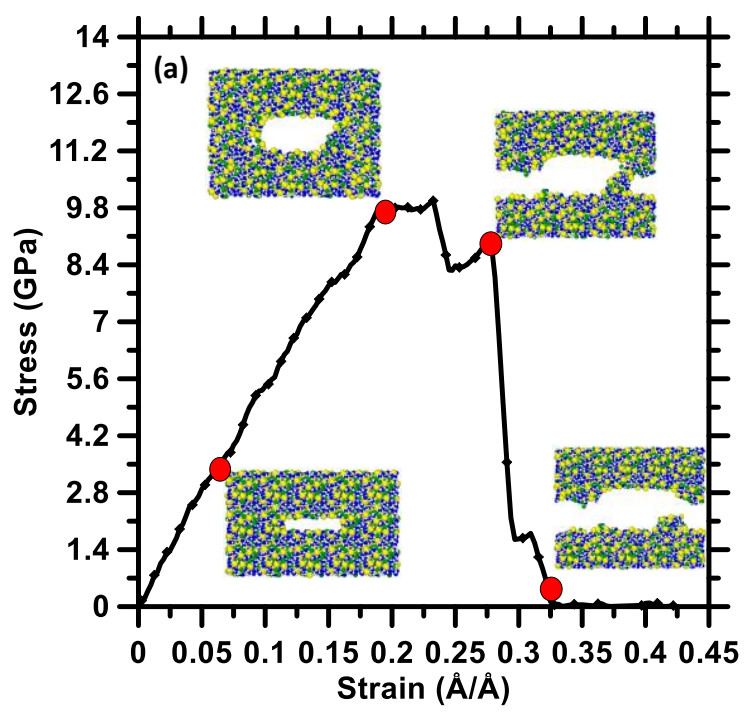

Figure 7. Tensile stress-strain response of the NAS structure with initial flaw

The maximum stress for NAS glass is observed as $9.8 \mathrm{GPa}$ (approx.) at a strain of $18 \%$. As the strain is increased beyond $18 \%$, small amount of ductility is observed. Similar behavior is observed and reported 
in the literature in the case of sodium silicate (NS) glass, and Calcium aluminosilicate (CAS) glass ${ }^{34}$. Oxide glasses also have been shown to exhibit small amount of plastic deformation ${ }^{67}$, which is needed to be taken in consideration during numerical simulation to capture such irreversible plasticity behavior efficiently. As per Dugdale-Barenblatt formula ${ }^{68-70}$, an estimation of the length of plasticity zone $r_{p l}$ is expressed as:

$$
r_{p l}=\frac{\pi}{8}\left(\frac{K_{I C}}{\sigma_{p l}}\right)^{2}
$$

where $\sigma_{p l}$ is the plastic yield stress of the material. Considering the influence of plasticity, the effective area of crack can be further modified $a s^{55}$ :

$$
\Delta A_{\text {eff }}=\Delta A-\frac{L_{z} r_{p l}}{2}
$$

This correction also addressed the issue of overlapping of the plastic zones at the periodic boundaries due to periodic condition applied in all directions. Similar methodology has also been adopted in the literature for silica glass ${ }^{34,71}$ and Calcium Silicate Hydrate $(\mathrm{CSH})^{44}$. The $r_{p l}$ for NAS glass is obtained as $7.2 \AA$ which is higher than that of quartz $(3.4 \AA)^{44}$. The $K_{I C}$ is then computed by using Equation 8 . The simulated value of $K_{I C}$ for NAS, thus obtained, is $0.8 \pm 0.05 \mathrm{MPa} \mathrm{m} \mathrm{m}^{0.5}$ which shows good correlation with the experimental value of $0.90 \pm 0.03 \mathrm{MPa} \mathrm{m}^{0.5}$, reported by Wiederhorn ${ }^{72}$. Such excellent match between the simulated and experimental fracture toughness of NAS glass signifies the efficacy of the adopted methodology towards efficient prediction of fracture toughness of similar systems. Hereafter, the validated simulation methodology is applied towards prediction of fracture toughness of $\mathrm{N}-\mathrm{A}-\mathrm{S}-\mathrm{H}$ with varying water content. 


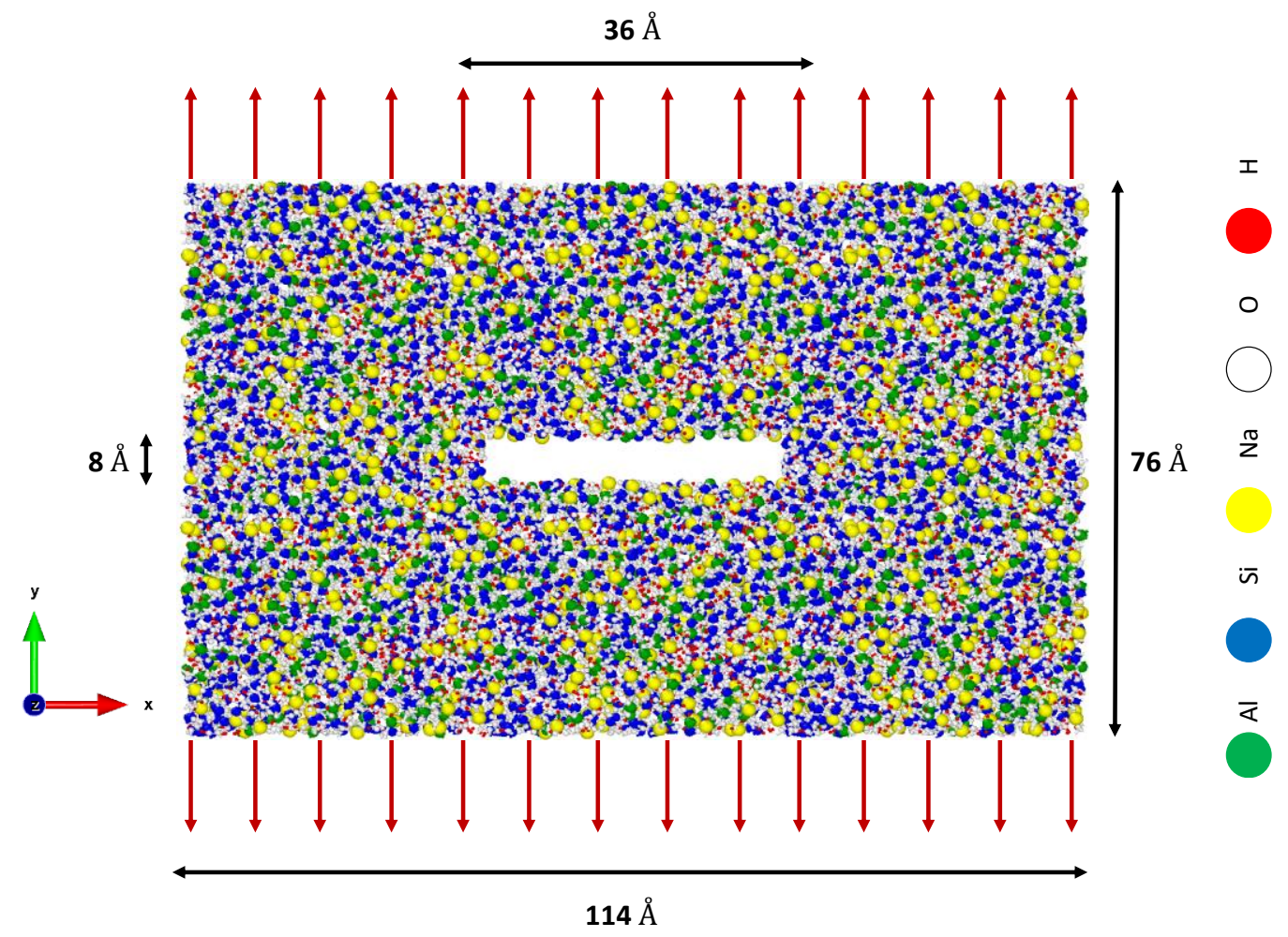

Figure 8. N-A-S-H structure with initial crack and loading direction along $y$-axis. The dimension of the box is $114 \AA$ x $76 \AA ̊$ x $38 \AA$ and initial crack size is $36 \AA$ x $8 \AA$ x $38 \AA$ along $\mathrm{x}, \mathrm{y}$ and $\mathrm{z}$ axes respectively.

Figure 8 shows a representative N-A-S-H structure (NASH15) with initial crack. Although the structure is shown for NASH15, the analysis is performed for all the structures with varying water content considered here. Here, size of the bounding box containing the molecular structure is $114 \AA$ x $76 \AA$ x $37 \AA$, obtained from sensitivity study as explained earlier for NAS glass. The size of the crack considered here is $36 \AA ̊$ x 8 $\AA$ x $37 \AA$. Such flaw size, relative to the size of the representative bounding box, has been successfully adopted towards prediction of fracture behavior of silicate glass and $\mathrm{CSH}^{34,44}$. Besides, as explained earlier, the fracture energy obtained using the present methodology has been shown to be insensitive to initial flaw size and box size as shown in $^{55,71}$. The flaw-construction procedure is same as explained earlier for NAS glass.

Under applied uniaxial tensile strain, the stress response for the fracture simulation for all the N-A-S-H structures are shown in Figure 9 along with the state of crack propagation at different stages. 

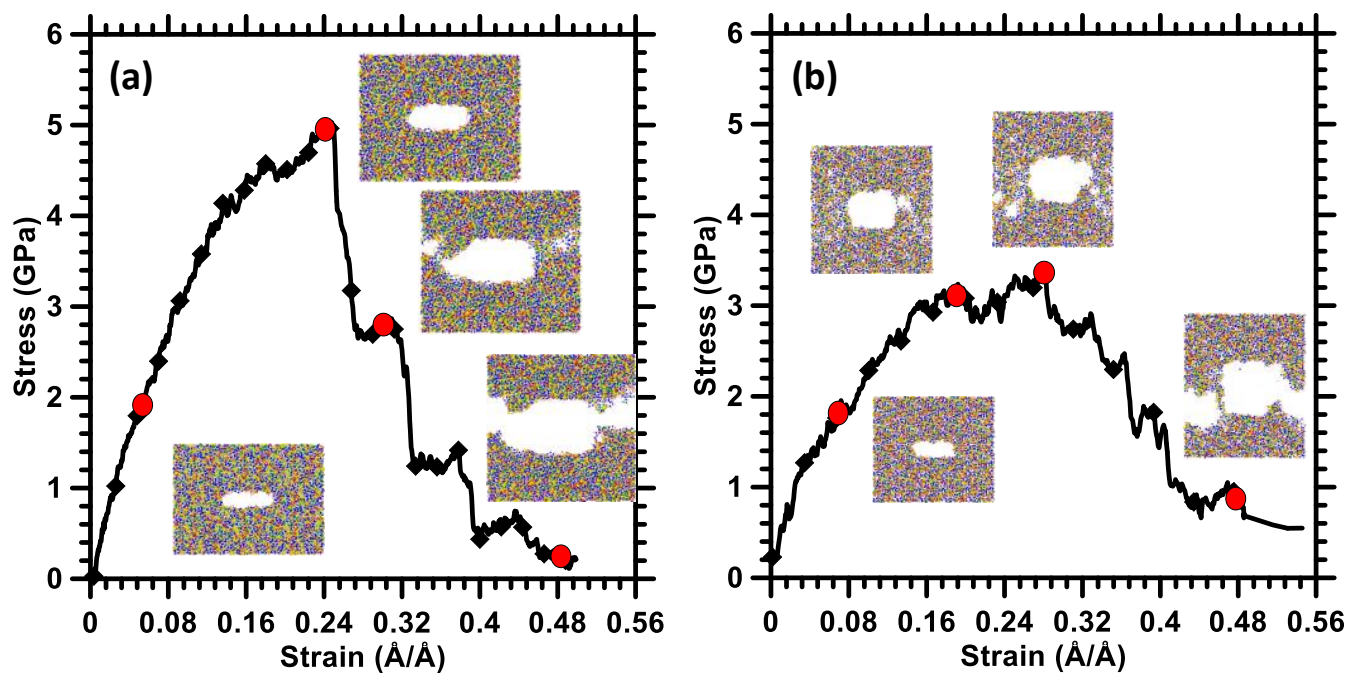

Figure 9. Tensile stress-strain response of the N-A-S-H structure with initial flaw for: (a) $15 \%$ water content, and (b) $20 \%$ water content.

Similar to NAS glass, N-A-S-H structure also shows mild plastic behavior which resists sudden failure of structure after reaching the peak stress. Similar observation is also reported for CSH by Bauchy et al. ${ }^{44}$ With increasing water content, the maximum stress reduces significantly which can be attributed to reduced polymerization in N-A-S-H due to the formation of more Al-O-H, Si-O-H bonds, and thus increasing the non-bridging oxygen (NBO) with increasing water content (as shown in Figure 5). Also, the slight increase in plastic behavior in the post-peak regime is also observed with increase in water as can be seen clearly from Figure 9. Form the tensile stress-strain responses, the fracture toughness is calculated for all the N-A-S-H structures with varying water content and the values are shown in Table 2. It is clearly observed that increase in water content in the N-A-S-H structure results in decrease in fracture toughness due to the reasons explained earlier in this section. The results obtained from MD simulation lies between the fracture toughness of glass structure and that of $\mathrm{CSH}$. For direct experimental validation, to the best of our knowledge, no experimental measurement of fracture toughness of N-A-S-H is currently available. However, the simulated values can be compared with available experimental values of fracture toughness of fly ash-based geopolymer mortar and geopolymer concrete. While experimentally obtained fracture toughness of fly ash-based geopolymer mortar has been reported to be in the range of $0.25-0.47$ $M P a m^{0.573,74}$, the values in the range of 0.6-0.9 $M P a m^{0.515}$ for fracture toughness of geopolymer concrete have been experimentally obtained. Although, we need to keep in mind that geopolymer mortar and concrete are complex heterogeneous systems containing multiple phases such as $\mathrm{N}-\mathrm{A}-\mathrm{S}-\mathrm{H}$, pores, unreacted fly ash, partially activated fly ash, fly ash with cavities, coarse aggregates, fine aggregates and such heterogeneity prevents direct experimental validation of the simulated fracture toughness of N-A-S- 
$\mathrm{H}$ despite apparent agreement. However, the simulated values are of the same order as experimentally observed for fly ash-based geopolymer mortars/concretes which fortifies the ability of the current simulations to yield realistic fracture toughness for N-A-S-H.

Table 2: Fracture toughness of $\mathrm{N}-\mathrm{A}-\mathrm{S}-\mathrm{H}$ with varying water content

\begin{tabular}{|c|c|c|c|}
\hline \multicolumn{4}{|c|}{ Simulated $K_{I C}\left(\mathrm{MPa} \mathrm{m}^{0.5}\right)$} \\
\hline NASH15 & NASH20 & NAS & $\mathrm{CSH}^{44}$ \\
\hline $0.45 \pm 0.03$ & $0.40 \pm 0.02$ & $0.80 \pm 0.05$ & $0.369 \pm 0.03$ \\
\hline
\end{tabular}

Table 3 represents the radius of plasticity zone $r_{p l}$ for NASH with different water content. It is observed that with increase in water content $r_{p l}$ also increases, which is evident from the post-peak stress-strain responses shown in Figure 9. The value obtained is smaller than that of $\mathrm{CSH}$, but lower than that of kerogen $(19.9 \AA)^{55}$. In this study, the brittleness/plastic behavior of different structures has also been computed and shown in Table 3. The brittleness index (B) is calculated following the procedure detailed in the literature ${ }^{44}$. The brittleness index is expressed as $\mathrm{B}=2 \gamma_{s} / G_{C}$. The term $\gamma_{s}$ refers to the surface energy. The obtained brittleness index values are shown in Table 3. The brittleness index for N-A-S-H is found to be lower than that reported for $\mathrm{C}-\mathrm{S}-\mathrm{H}^{44}$, suggesting a more ductile deformation in the $\mathrm{N}-\mathrm{A}-\mathrm{S}-\mathrm{H}$. To shed more light on the influence of the molecular structure on the crack propagation behavior, the forthcoming section evaluates change in different pair atoms in N-A-S-H structure during propagation of crack for detailed fundamental insights.

Table 3: Brittleness parameter and Length of $r_{p l}$ of $\mathrm{N}-\mathrm{A}-\mathrm{S}-\mathrm{H}$ with varying water content

\begin{tabular}{|c|c|c|c|c|c|}
\hline \multicolumn{3}{|c|}{ Simulated brittleness index $(B)$} & \multicolumn{3}{c|}{ Simulated $r_{p l}(\AA)$} \\
\hline NASH15 & NASH20 & CSH $^{44}$ & NASH15 & NASH20 & CSH $^{44}$ \\
\hline 0.49 & 0.40 & 0.62 & 16.40 & 17.50 & 13.7 \\
\hline
\end{tabular}

\subsection{Assessment on the nature of pair atoms during fracture}

This section reports the change in number of bonds with increasing strain during the tensile simulation for the representative NASH15 structure. The cutoff distances for various pair atom interactions are judiciously chosen as the first minimum after the first peak of the partial PDFs to obtain representative assessments. The partial PDFs for $\mathrm{Na}-\mathrm{X}$ pairs (X: $\mathrm{Al}, \mathrm{Si}, \mathrm{Na}, \mathrm{O}, \mathrm{Ow}, \mathrm{H} ; \mathrm{Ow}$ refers to oxygen in water) and 
chosen cutoff distances for all the pair atom interactions are provided in the supplementary document. Here, the negative sign represents decrease in the number of bonds, which can be correlated with either breakage of bonds or elongation of bonds and the positive sign indicates increase in number of bonds. Figure 10(a-d) shows the variation for different type of bonds.
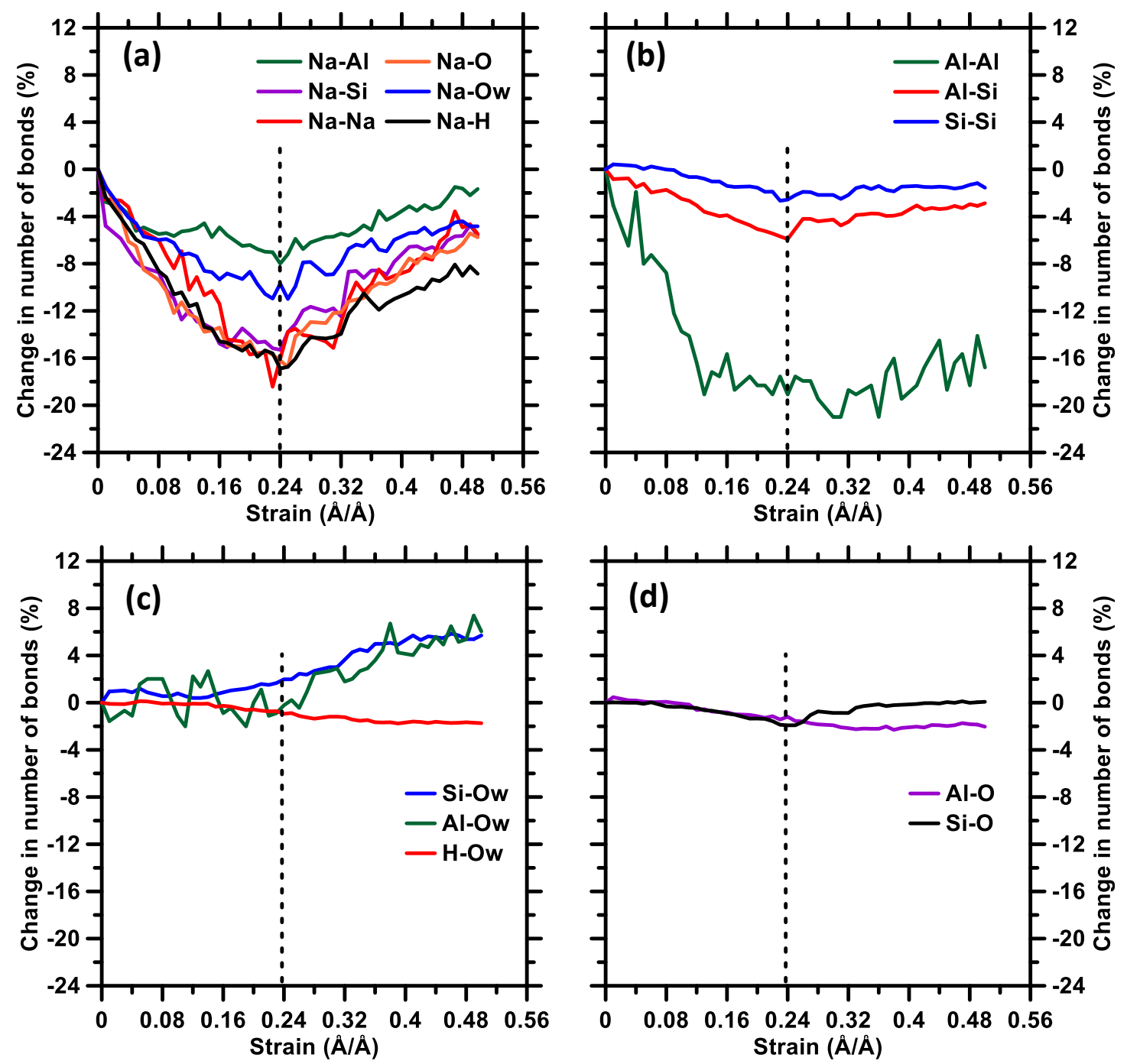

Figure 10. Change in pair atom interactions with varying strain: (a) $\mathrm{Na-X}(\mathrm{X}: \mathrm{Al}, \mathrm{Si}, \mathrm{Na}, \mathrm{O}, \mathrm{Ow}, \mathrm{H}),(\mathrm{b}) \mathrm{Al}-\mathrm{Al}$, $\mathrm{Al}-\mathrm{Si}, \mathrm{Si}-\mathrm{Si}$ (c) X-Ow (X: Al, Si), and (d) X-O (X: Al, Si). Ow refers to oxygen in water

It is observed that the ionic bonds (in particular atoms paired with sodium ( $\mathrm{Na}-\mathrm{X}, \mathrm{X}=\mathrm{Al}, \mathrm{Si}, \mathrm{O}, \mathrm{Ow}, \mathrm{H}$ )) show anomalous behavior (shown in Figure 10(a)) during the elongation due to lower amount of energy needed to extend or break such bonds (bond dissociation energy) as compared to covalent bonds. The general trend in $\mathrm{Na}-\mathrm{X}$ pairs $(\mathrm{X}=\mathrm{Al}, \mathrm{Si}, \mathrm{O}, \mathrm{Ow}, \mathrm{H})$ during fracture suggests that the number of $\mathrm{Na}-\mathrm{X}$ bonds decreases with increasing strain during the pre-peak stage till the peak stress is reached beyond which the trend reverses with gradual increase in number of $\mathrm{Na}-\mathrm{X}$ bonds with increasing strain in the post-peak 
regime (indicates the contribution of sodium towards plastic region) leading to failure. The global minima in the change in the number of $\mathrm{Na}-\mathrm{X}$ bonds-strain response corresponds to the yield strain (corresponding to peak stress) observed during the tensile simulation. Similar observations are reported in the literature ${ }^{75}$ for silicate glasses wherein increasing number of $\mathrm{Na}-\mathrm{X}$ bonds beyond the peak stress has been associated with plastic deformations in the post-peak regime.

The interactions of sodium ions with the Si skeleton in silicate glasses ${ }^{75}$ are very complex because of high mobility of the sodium ions, thereby inducing local softening ${ }^{76}$, but at the same time their presence has been shown to prevent the isotropic collapse of Si rings ${ }^{75}$. This is also being observed here for the Si-Si interactions where change in the number of pair atoms with increasing strain is relatively insignificant (Figure 10(b)). For the case of Al-Al and Al-Si interactions, the nature of decreasing curve can be well correlated with the bond dissociation energy, where the orders of bond dissociation energy are Al-Al<Al$\mathrm{Si}<\mathrm{Si}^{-\mathrm{Si}^{77}}$. Due to low bond dissociation energy associated with Al-Al pairs, significant reduction in the number of Al-Al interactions are observed whereas such significant reduction in interactions are not observed for Al-Si pairs.

The system becomes more complex with the presence of water, as it also contributes towards significant increase in the number of $\mathrm{NBO}^{78,79}$ and consequently, it leads to decrease in polymerization of the structure $^{44}$. During the elongation, nanovoids are expanded ${ }^{49}$ allowing water to diffuse into the ring structure. This results in the formation of $\mathrm{X}-\mathrm{OW}-\mathrm{H}$ bonds (X: Si, Al and $\mathrm{Ow}$ indicates oxygen connected to water), which is also evident from the increase in the number of Si-Ow and Al-Ow bonds as shown in Figure 10(c). In the case of $\mathrm{H}$-Ow pairs, the number of available bonds decreases with increase in strain. This specify the effect of dissociation of water where the $\mathrm{H}-\mathrm{O} w-\mathrm{H}$ dissociates into $\mathrm{OW}-\mathrm{H}$ and $\mathrm{H}$ ions. Similar observations are reported in case of C-S-H as well ${ }^{44}$. The Al-O and Si-O pairs (Figure 10(d)) show relatively smaller changes in number of bonds. This suggests that the crack propagation is facilitated by breakage of ionic bonds and dissociation of water rather than breakage of strong $\mathrm{Al}-\mathrm{O}$ and Si-O network structure.

\section{CONCLUSION}

This paper presents MD simulation-based evaluation of the fracture toughness of the N-A-S-H gel in fly ash based geopolymers. For MD simulations, the molecular structures of $\mathrm{N}-\mathrm{A}-\mathrm{S}-\mathrm{H}$ are constructed using melt-quench method followed by adsorption of water in the structure using GCMC. The simulations are performed using ReaxFF potential. The total pair distribution peaks and the structure factor peaks for the constructed structures (especially the structures containing up to $20 \%$ water) matches closely with experimental neutron diffraction data signifying good correlation in the short- and medium-range order 
of the structures. The simulated values of fracture toughness of N-A-S-H appears to be very close to that of fly ash-based geopolymer mortar and the simulated fracture toughness values for $\mathrm{N}-\mathrm{A}-\mathrm{S}-\mathrm{H}$ are found to be of the same order as experimentally observed fracture toughness of geopolymer concrete. This suggests that N-A-S-H plays a key role in the fracture response of fly ash-based geopolymers and it strengthens the efficacy of the molecular dynamics simulation towards obtaining realistic fracture toughness of $\mathrm{N}-\mathrm{A}-\mathrm{S}-\mathrm{H}$ which is otherwise challenging to obtain experimentally. To shed more light on the influence of different bonds in the N-A-S-H structure on its fracture response, a state of different bonds during the entire tensile simulation process is reported. The general trends suggest that the $\mathrm{Na}-\mathrm{X}(\mathrm{X}: \mathrm{Al}$, $\mathrm{Si}, \mathrm{O}, \mathrm{Ow}, \mathrm{H}$ ) pairs shows significant interaction with the propagation of crack in the N-A-S-H structure under increasing applied uniaxial tensile strain due to their lower bond dissociation energy whereas strong covalent bonds in the N-A-S-H structures shows relatively lower sensitivity towards propagation of crack with increasing strain. Such fundamental insights involving the influence of different bond pairs on the crack propagation mechanisms in N-A-S-H atomic structure can captivate development of fundamental strategies in the future to tune the molecular structure of $\mathrm{N}-\mathrm{A}-\mathrm{S}-\mathrm{H}$ for improved toughness. Overall, the atomic scale simulation methodology, presented in this study, to obtain fracture toughness of N-A-S-H open up avenues towards multiscale numerical simulation-based design of fly ash based geopolymer binders in bottom-up approach for enhanced performance.

\section{SUPPLEMENTARY MATERIAL}

See supplementary material for chosen cutoff distances for all the pair atom interactions and Na-X (X: Al, $\mathrm{Si}, \mathrm{Na}, \mathrm{O}, \mathrm{Ow}, \mathrm{H}$; Ow refers to oxygen in water) partial PDFs towards assessment on the nature of pair atoms during fracture.

\section{REFERENCES}

${ }^{1}$ P. Wray and K. Scrivener, American Ceramic Society Bulletin 91, 47 (2012).

${ }^{2}$ P.K. Mehta, Concr. Int. 23, 61 (2001).

${ }^{3}$ G. Habert, J.B. d'Espinose de Lacaillerie, and N. Roussel, Journal of Cleaner Production 19, 1229 (2011).

${ }^{4}$ S. Das, B. Souliman, D. Stone, and N. Neithalath, ACS Appl. Mater. Interfaces 6, 8295 (2014).

${ }^{5}$ J. Davidovits, Journal of Thermal Analysis 37, 1633 (1991).

${ }^{6}$ A. Fernandez-Jimenez and A. Palomo, ACI Mater J 103, 106 (2006).

${ }^{7}$ P. Duxson, A. Fernández-Jiménez, J.L. Provis, G.C. Lukey, A. Palomo, and J.S.J. van Deventer, J Mater Sci 42, 2917 (2007).

${ }^{8}$ T. Bakharev, J.G. Sanjayan, and Y.-B. Cheng, Cement and Concrete Research 29, 113 (1999).

${ }^{9}$ J.L. Provis, A. Palomo, and C. Shi, Cement and Concrete Research 78, 110 (2015). 
${ }^{10}$ J.L. Provis and J.S.J. van Deventer, Alkali Activated Materials: State-of-the-Art Report, RILEM TC 224-AAM (Springer Science \& Business Media, 2013).

11 J.L. Provis and S.A. Bernal, Annual Review of Materials Research 44, 299 (2014).

12 S. Das, P. Yang, S.S. Singh, J.C.E. Mertens, X. Xiao, N. Chawla, and N. Neithalath, Cement and Concrete Research 78, 252 (2015).

${ }^{13}$ S. Nayak, A. Kizilkanat, N. Neithalath, and S. Das, Journal of Materials in Civil Engineering 31, 04019043 (2019).

14 B.C. McLellan, R.P. Williams, J. Lay, A. van Riessen, and G.D. Corder, Journal of Cleaner Production 19, 1080 (2011).

${ }^{15}$ P.K. Sarker, R. Haque, and K.V. Ramgolam, Materials \& Design 44, 580 (2013).

${ }^{16}$ Y. Lu, J Civil Environment Engg 01, (2012).

${ }^{17}$ A. Fernández-Jiménez and A. Palomo, Cement and Concrete Research 35, 1984 (2005).

18 T. Bakharev, Cement and Concrete Research 35, 1224 (2005).

${ }^{19}$ K.E. Drexler, C. Peterson, and G. Pergamit, in William Morrow (New York, 1991).

${ }^{20}$ M.R. Sadat, S. Bringuier, A. Asaduzzaman, K. Muralidharan, and L. Zhang, J. Chem. Phys. 145, 134706 (2016).

${ }^{21}$ D. Hou, Y. Zhang, T. Yang, J. Zhang, H. Pei, J. Zhang, J. Jiang, and T. Li, Physical Chemistry Chemical Physics 20, 20695 (2018).

${ }^{22}$ F. Lolli, H. Manzano, J.L. Provis, M.C. Bignozzi, and E. Masoero, ACS Appl. Mater. Interfaces 10, 22809 (2018).

${ }^{23}$ G.A. Lyngdoh, R. Kumar, N.M.A. Krishnan, and S. Das, J. Chem. Phys. 151, 064307 (2019).

${ }^{24}$ M. Zhang, N.A. Deskins, G. Zhang, R.T. Cygan, and M. Tao, J. Phys. Chem. C 122, 6760 (2018).

25 J.L. Provis and J.S.J. van Deventer, Geopolymers: Structures, Processing, Properties and Industrial Applications (Elsevier, 2009).

${ }^{26}$ J. Puibasset and R.J.-M. Pellenq, The Journal of Physical Chemistry B 112, 6390 (2008).

27 A.C.T. van Duin, S. Dasgupta, F. Lorant, and W.A. Goddard, J. Phys. Chem. A 105, 9396 (2001).

${ }^{28}$ C.E. White, J.L. Provis, T. Proffen, and J.S.J. Van Deventer, Journal of the American Ceramic Society 93, 3486 (2010).

${ }^{29}$ C.E. White, J.L. Provis, A. Llobet, T. Proffen, and J.S.J. van Deventer, Journal of the American Ceramic Society 94, 3532 (2011).

${ }^{30}$ C.E. White, J.L. Provis, T. Proffen, D.P. Riley, and J.S.J. van Deventer, Physical Chemistry Chemical Physics 12, 3239 (2010).

${ }^{31}$ C.E. White, J.L. Provis, B. Bloomer, N.J. Henson, and K. Page, Physical Chemistry Chemical Physics 15, 8573 (2013).

32 J.L. Provis, A. Hajimohammadi, C.E. White, S.A. Bernal, R.J. Myers, R.P. Winarski, V. Rose, T.E. Proffen, A. Llobet, and J.S.J. van Deventer, Cement and Concrete Composites 36, 56 (2013).

${ }^{33}$ S. Plimpton, Journal of Computational Physics 117, 1 (1995).

${ }^{34}$ Y. Yu, B. Wang, Y.J. Lee, and M. Bauchy, MRS Proceedings 1757, mrsf14 (2015).

${ }^{35}$ M. Bauchy, The Journal of Chemical Physics 141, 024507 (2014).

${ }^{36}$ M. Bauchy, B. Wang, M. Wang, Y. Yu, M.J. Abdolhosseini Qomi, M.M. Smedskjaer, C. Bichara, F.-J. Ulm, and R. Pellenq, Acta Materialia 121, 234 (2016). 
${ }^{37}$ X. Li, W. Song, K. Yang, N.M.A. Krishnan, B. Wang, M.M. Smedskjaer, J.C. Mauro, G. Sant, M. Balonis, and M. Bauchy, The Journal of Chemical Physics 147, 074501 (2017).

${ }^{38}$ L. Ly, E.R. Vance, D.S. Perera, Z. Aly, and K. Olufson, Advances in Technology of Materials and Materials Processing Journal 8, 236 (2006).

${ }^{39}$ M. Lizcano, A. Gonzalez, S. Basu, K. Lozano, and M. Radovic, Journal of the American Ceramic Society 95, 2169 (2012).

${ }^{40}$ H.M. Aktulga, J.C. Fogarty, S.A. Pandit, and A.Y. Grama, Parallel Computing 38, 245 (2012).

${ }^{41}$ D. Frenkel and B. Smit, Understanding Molecular Simulation: From Algorithms to Applications (Elsevier, 2001).

${ }^{42}$ P. Duxson, G.C. Lukey, and J.S.J. van Deventer, Journal of Materials Science 42, 3044 (2007).

${ }^{43}$ N.M.A. Krishnan, B. Wang, Y. Le Pape, G. Sant, and M. Bauchy, Physical Review Materials 1, (2017).

${ }^{44}$ M. Bauchy, H. Laubie, M.J. Abdolhosseini Qomi, C.G. Hoover, F.-J. Ulm, and R.J.-M. Pellenq, Journal of Non-Crystalline Solids 419, 58 (2015).

${ }^{45}$ N.M.A. Krishnan, R. Ravinder, R. Kumar, Y. Le Pape, G. Sant, and M. Bauchy, Acta Materialia 166, 611 (2019).

${ }^{46}$ M. Bauchy, The Journal of Chemical Physics 137, 044510 (2012).

${ }^{47}$ A.C. Wright, Journal of Non-Crystalline Solids 106, 1 (1988).

48 J. Du and L.R. Corrales, Journal of Non-Crystalline Solids 352, 3255 (2006).

${ }^{49}$ M.R. Sadat, S. Bringuier, K. Muralidharan, K. Runge, A. Asaduzzaman, and L. Zhang, Journal of Non-Crystalline Solids 434, 53 (2016).

${ }^{50}$ S.C. Chowdhury, B.Z. (Gama) Haque, and J.W. Gillespie, J Mater Sci 51, 10139 (2016).

${ }^{51}$ M.R. Sadat, S. Bringuier, K. Muralidharan, G. Frantziskonis, and L. Zhang, Computational Materials Science 142, 227 (2018).

52 D. Hou, J. Zhang, Z. Li, and Y. Zhu, Mater Struct 48, 3811 (2015).

53 R. Clausius, The London, Edinburgh, and Dublin Philosophical Magazine and Journal of Science 40, 122 (1870).

${ }^{54}$ D.H. Tsai, J. Chem. Phys. 70, 1375 (1979).

55 Brochard Laurent, Hantal Gyorgy, Laubie Hadrien, Ulm Franz J., and Pellenq Roland J. -M., Poromechanics V 2471 (n.d.).

56 Griffith Alan Arnold and Taylor Geoffrey Ingram, Philosophical Transactions of the Royal Society of London. Series A, Containing Papers of a Mathematical or Physical Character 221, 163 (1921).

${ }^{57}$ J.-B. Leblond, Comptes Rendus de l'Académie Des Sciences - Series IIB - Mechanics-PhysicsChemistry-Astronomy 326, 243 (1998).

58 T.L. Anderson, Fracture Mechanics: Fundamentals and Applications (CRC Press/Taylor \& Francis, 2005).

${ }^{59}$ B. Wang, Y. Yu, Y.J. Lee, and M. Bauchy, Front. Mater. 2, (2015).

${ }^{60}$ G.R. Irwin, in Elasticity and Plasticity / Elastizität Und Plastizität, edited by S. Flügge (Springer Berlin Heidelberg, Berlin, Heidelberg, 1958), pp. 551-590.

${ }^{61}$ A.C. Wright, Journal of Non-Crystalline Solids 159, 264 (1993).

${ }^{62}$ Y. Xiang, J. Du, M.M. Smedskjaer, and J.C. Mauro, The Journal of Chemical Physics 139, 044507 (2013). 
${ }^{63}$ L. Brochard, G. Hantal, H. Laubie, F.-J. Ulm, and R.J.M. Pellenq, Int J Fract 194, 149 (2015).

${ }^{64}$ R. Ravinder, A. Kumar, R. Kumar, P. Vangla, and N.M.A. Krishnan, Journal of the American Ceramic Society $\mathbf{n} / \mathbf{a}$, (n.d.).

${ }^{65}$ S. Nosé, Molecular Physics 52, 255 (1984).

${ }^{66}$ W.G. Hoover, Phys. Rev. A 31, 1695 (1985).

${ }^{67}$ F. Célarié, S. Prades, D. Bonamy, L. Ferrero, E. Bouchaud, C. Guillot, and C. Marlière, Phys. Rev. Lett. 90, 075504 (2003).

${ }^{68}$ G.I. Barenblatt, in Advances in Applied Mechanics, edited by H.L. Dryden, Th. von Kármán, G. Kuerti, F.H. van den Dungen, and L. Howarth (Elsevier, 1962), pp. 55-129.

${ }^{69}$ J.M. Lemm, in (New York: Academic Press, 1962).

${ }^{70}$ D.S. Dugdale, Journal of the Mechanics and Physics of Solids 8, 100 (1960).

${ }^{71}$ R. Ravinder, A. Kumar, R. Kumar, P. Vangla, and N.M.A. Krishnan, J Am Ceram Soc 1 (2019).

${ }^{72}$ S.M. Wiederhorn, Journal of the American Ceramic Society 52, 99 (1969).

73 T. Phoo-ngernkham, V. Sata, S. Hanjitsuwan, C. Ridtirud, S. Hatanaka, and P. Chindaprasirt, Arab J Sci Eng 41, 1263 (2016).

${ }^{74}$ P. Rovnaník, H. Šimonová, L. Topoláŕ, P. Schmid, and Z. Keršner, Procedia Engineering 151, 321 (2016).

${ }^{75}$ G. Molnár, P. Ganster, and A. Tanguy, Phys. Rev. E 95, 043001 (2017).

${ }^{76}$ Y. Vaills, T. Qu, M. Micoulaut, F. Chaimbault, and P. Boolchand, J. Phys.: Condens. Matter 17, 4889 (2005).

77 Y.-R. Luo, Handbook of Bond Dissociation Energies in Organic Compounds (CRC Press, 2002).

${ }^{78}$ T. Ohkubo, S. Gin, M. Collin, and Y. Iwadate, Scientific Reports 8, (2018).

${ }^{79}$ Y. Zhang, J. Zhang, J. Jiang, D. Hou, and J. Zhang, Construction and Building Materials 193, 491 (2018). 